\title{
An infrequent association: Left ventricular non compaction with bicuspid aortic valve and coarctation of the aorta.
}

\author{
Aureo Campos-Tello ${ }^{*}$, Giancarlo A Valle ${ }^{1}$, Félix A Revilla ${ }^{1}$, Sara Ramírez-Flores², Ángel Cueva- \\ Parra $^{1}$, Zoila Rodríguez-Urteaga ${ }^{3}$
}

${ }^{1}$ Department of Cardiology, Hospital Nacional Dos de Mayo, Lima, Peru

${ }^{2}$ Department of Cardiology, Hospital Central FAP, Lima, Peru

${ }^{3}$ Department of Cardiology, Instituto Nacional Cardiovascular INCOR, Lima, Peru

\section{Abstract}

Left ventricular non compaction (LVNC) is a condition rarely diagnosed and reported in current literature. It affects approximately 8 to 12 per 1 million of individuals and its causes are not well understood, but they are linked to gene mutations. The authors present a 29-year-old male who came to medical consult for a pre surgical evaluation. He was completely asymptomatic. Due to an aortic diastolic murmur we performed a transthoracic echocardiogram which revealed severe aortic regurgitation, signs of left ventricular non compaction and coarctation of the aorta. Subsequently, we decided to send him for a CT scan and CMR that allowed to confirm our diagnosis and gave more support to our findings. We're aimed to report this case because LVNC was found in a patient without symptoms but with a murmur missed in previous medical evaluations. Then, we encourage to be very accurate in patient's examination and use non- invasive methods properly in order to identify such important cases.

Kewords: Left ventricular non compaction, Bicuspid aortic valve, Coarctation of the aorta.

Accepted on Feb 20, 2020

\section{Introduction}

Left ventricular non compaction (LVNC) is a rare congenital cardiomyopathy that can be diagnosed at any age. This disease makes the LV to become "spongy" and have two separate layers: a compacted epicardial layer and a large non compacted endocardial layer with trabeculation and deep recesses communicated within them but not with coronary circulation. Its reported prevalence is between 0.014 and $1.3 \%$ according to echocardiographic studies [1].

Despite the exact mechanisms are not well comprehended, several gene mutations (especially in MYH7 and MYBPC3 genes) avoid myocardium to become smooth and firm (compacted) during intrauterine life [2].

This condition is usually asymptomatic, but can be complicated by heart failure, thromboembolism or ventricular arrhythmias, including sudden cardiac death. It's primarily diagnosed with echocardiography and confirmed with CMR [3].

In this manuscript, we report a case of LVNC associated with two other abnormalities: bicuspid aortic valve and coarctation of the aorta. This case is important because this congenital disease is underdiagnosed and its prompt recognition is necessary to avoid further complications.

\section{Case Presentation}

An asymptomatic 29-year-old male from Lima attended to a pre surgical evaluation for knee intervention, in our department. At physical exam, his vitals were: heart rate of 63 bpm, BP of 145/91 mmHg (measured in right arm), respiratory rate 14 breath/minute and blood oxygen saturation of $96 \%$. The cardiovascular exam revealed a diastolic murmur in aortic area and diminished pulse amplitude in lower limbs. Therefore, BP was measured in left leg finding $100 / 60 \mathrm{mmHg}$. Due to described murmur and BP difference between arms and legs, there was suspicion of aortic regurgitation and coarctation of the aorta.

Electrocardiography showed sinus rhythm, normal axis, signs of LV hypertrophy and pressure overload (Figure 1).

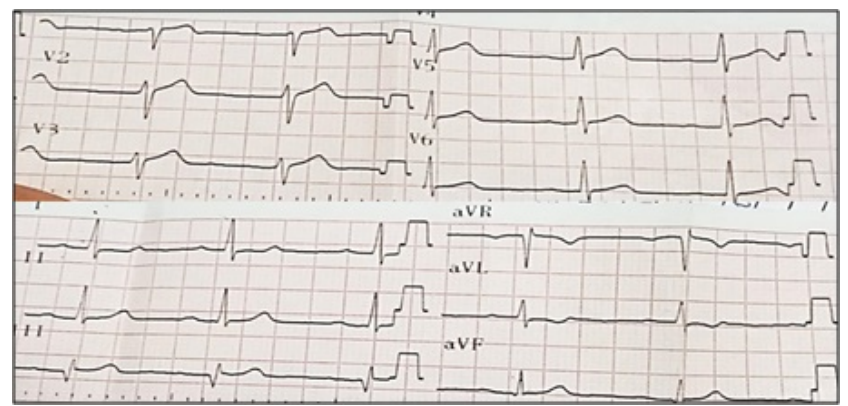

Figure 1. Electrocardiogram in sinus rhythm, LV hypertrophy and pressure overload.

The transthoracic echocardiography (TTE) showed the following findings:

1) In the PLAX view we identified LV with mild enlargement and moderate hypertrophy (end diastolic diameter: $57.5 \mathrm{~mm}$ and end systolic diameter: $37.2 \mathrm{~mm}$, IVSd: $13.5 \mathrm{~mm}$ ). The aorta was dilated at the sinuses of Valsalva level $(40.6 \mathrm{~mm})$. Also, the aortic valve had severe regurgitation with an eccentric jet (Figures 2A and 2B). 
Citation: Aureo Campos-Tello, Giancarlo A Valle, Félix A Revilla, et al. An infrequent association: Left ventricular non compaction with bicuspid aortic valve and coarctation of the aorta. Curr Trend Cardiol 2020;4(1):5-8.

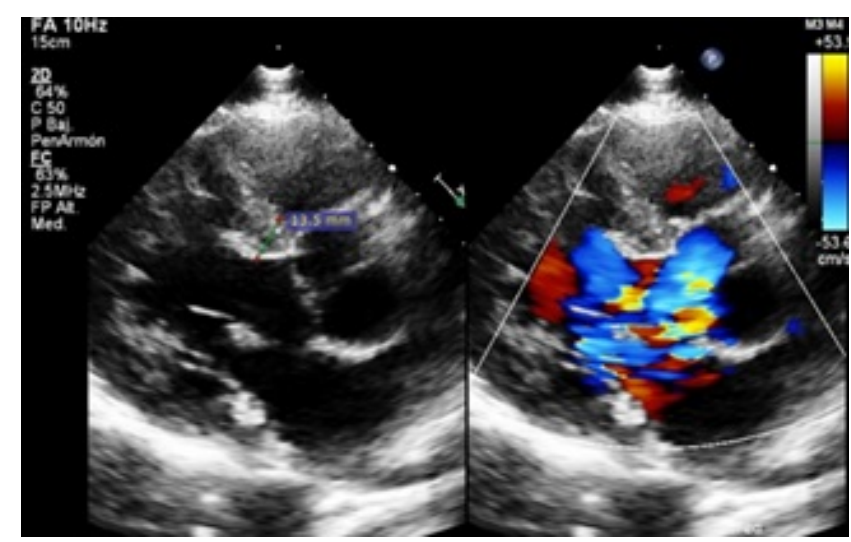

Figure 2A. In PLA X it's there's moderate LV hypertrophy and mild enlargement.

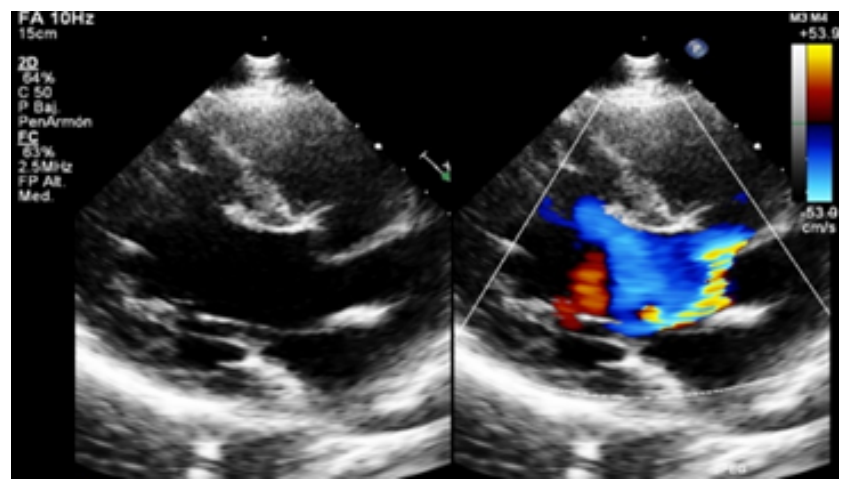

Figure 2B. In the Apical 4 chamber view blood flow within recesses is observed.

2) In the apical view it was possible to see blood flow within deep trabeculae and recesses (Figure 2C).

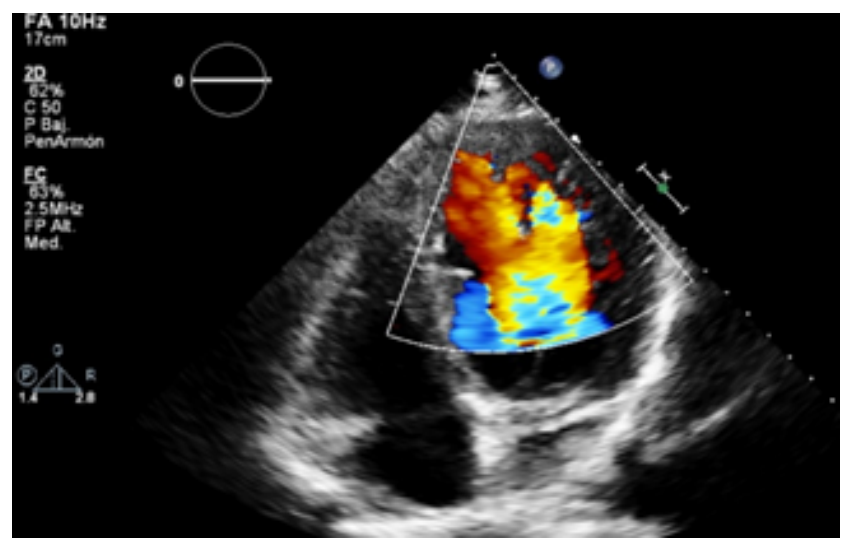

Figure 2C. Severe aortic regurgitation with an eccentric jet.

3) In the PSAX view the aortic valve seemed to be bicuspid due to fusion of LCC and RCC (anterior cusp in an AP phenotype). The NCC became the posterior cusp (Figure 2D).

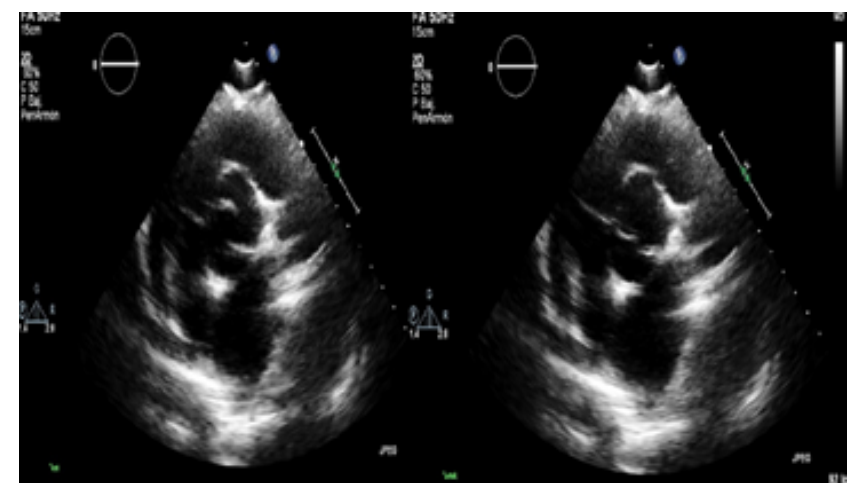

Figure 2D. Bicuspid aortic valve in systole and diastole.

4) In the suprasternal view, there was a narrowing in the descending thoracic aorta suggesting coarctation of the aorta. The CT confirmed the previous findings:

1) Hyper trabeculated LV and mild dilation of aorta at the sinuses of Valsalva level (42 mm) (Figures 3A-3D).
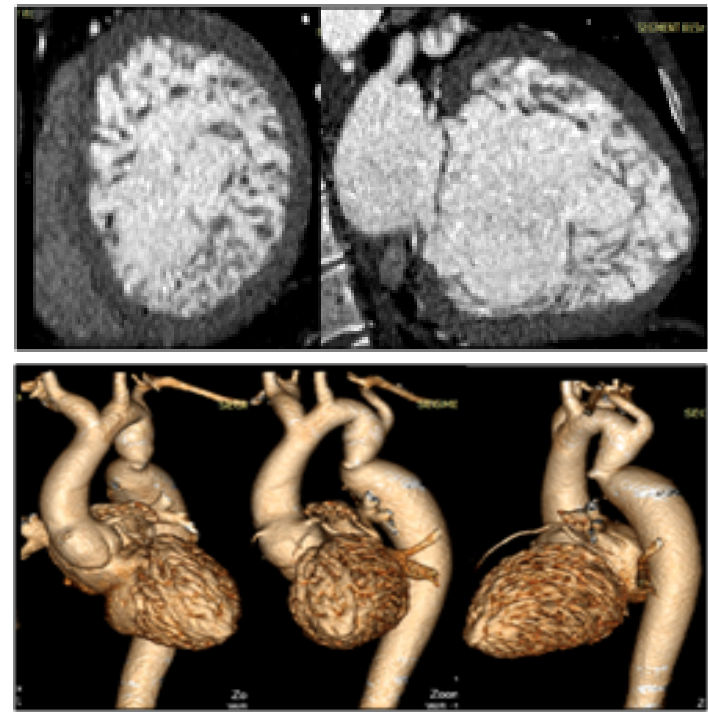

Figure 3A. CT showing hyper trabeculated, Volume rendering allows the visualization of dilated ascending aorta at sinuses of Valsalva level and coarctation of the thoracic descending aorta.

2) Bicuspid aortic valve (BAV) AP phenotype with fusion of LCC and RCC through a raphe (Figure 3B).

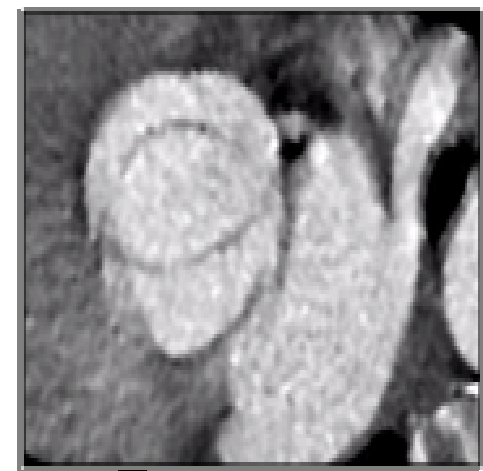

Figure 3B. Bicuspid aortic valve due to fusion of LCC and RCC (AP phenotype) C. Coarctation of the aorta.

3) The coarctation of the aorta measured $14 \times 19.5 \mathrm{~mm}$ with a $38 \%$ narrowing relative to the aortic diameter at the diaphragm level (Figures 3C and 3D). 


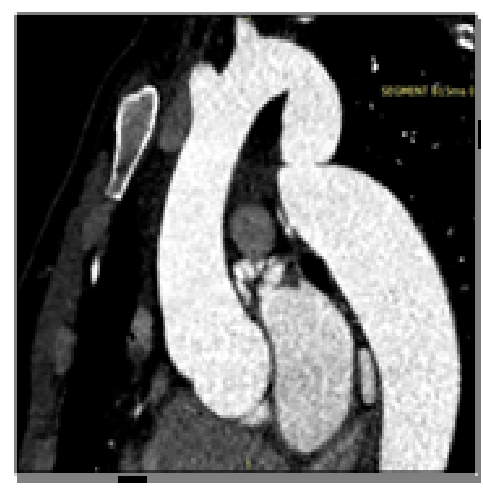

Figure 3C. Coarctation of the aorta.

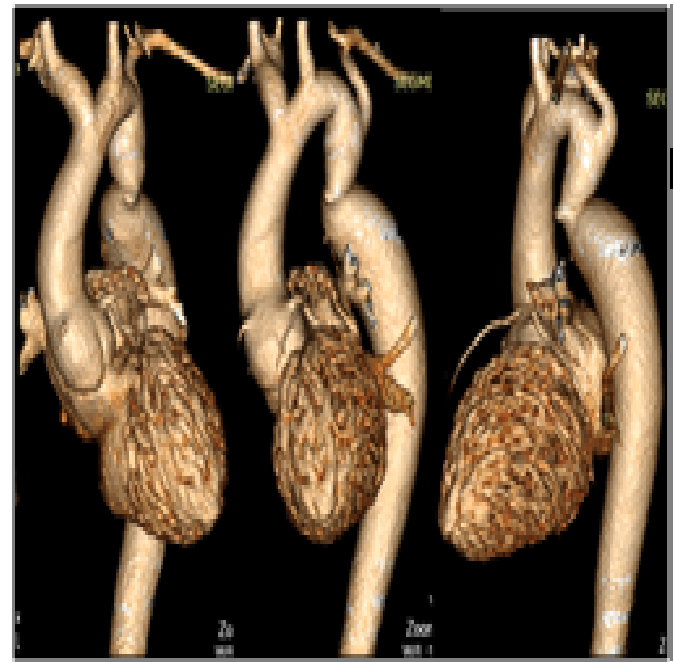

Figure 3D. Volume rendering allows the visualization of dilated ascending aorta at sinuses of Valsalva level and coarctation of the thoracic descending aorta.

The CMR allowed us to make our final diagnosis because:

1) The ratio of noncompacted myocardium to compacted myocardium was 3.8. However, there was no LGE (Figures 4 and 5).

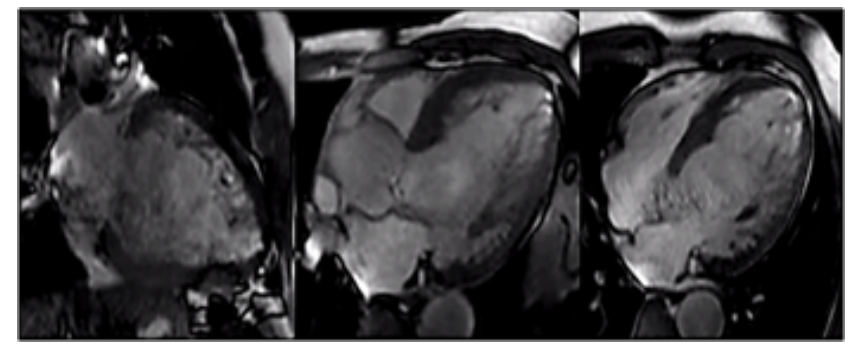

Figure 4. $C M R$ showing non-compacted myocardium in T1 sequences. The Petersen ratio $i .33$.

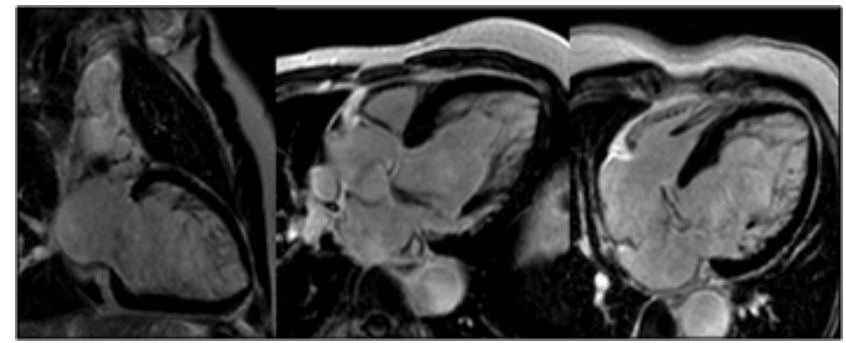

Figure 5. $C M R$ showing absence of $L G E$ meaning no evidence of fibrosis.

2) The LV ejection fraction was $65 \%$, its EDV was $277 \mathrm{ml} / \mathrm{m}^{2}$ (indexed $164 \mathrm{ml} / \mathrm{m}^{2}$ ) and $\mathrm{ESV}$ was $97 \mathrm{ml} / \mathrm{m}^{2}$ (indexed: 58 $\mathrm{ml} / \mathrm{m}^{2}$ ).

3) The maximum narrowing of the aorta at the coarctation was $12 \mathrm{~mm}$.

After all the described workup process we concluded: left ventricular non compaction associated with bicuspid aortic valve and coarctation of the aorta.

We performed an exercise test (Bruce protocol) and the patient reached 7 METS. It was stopped because he had knee pain.

\section{Discussion}

Left ventricular non compaction is an entity recognized for 80 years and is considered by European Society of Cardiology as a not classified familiar cardiomyopathy. Its presentation is isolated or associated with another abnormalities [1]. There are few reported cases associated with bicuspid aortic valve and coarctation of the aorta.

There are several criteria for diagnosis depending on the method used for that purpose. However, CMR has the best performance. Jacquier et al. described criteria for diagnosis of this disease: a trabecular LV mass above $20 \%$ of total mass has a sensitivity of $91.6 \%$ and a specificity of $86.5 \%$ [4]. Nevertheless, the most used criteria for diagnosis by CMR is the one described by Petersen et al.: the ratio of noncompacted myocardium to compacted myocardium must be greater than 2.3 during diastole [5]. Therefore, our patient met the criteria for diagnosis of LVNC because he had a Petersen ratio of 3.8 .

In current literature, there are some cases of LVNC with Ebstein's anomaly, but there are few associated with bicuspid aortic valve or coarctation of aorta. The first one (LVNC and BAV) was reported in 2003 when Cavusoglu et al. described a 19 year-old-male with heart failure due to a severely enlarged LV with moderate impairment in systolic function (LVEF: 35\%)[6]. In 2009, Wrigley et al. published another case of a 39 year-old-male with LV, BAV and aortic regurgitation, but the first one to undergo valve replacement surgery. $\mathrm{He}$ had a mildly dilated LV (end-diastolic diameter: $61 \mathrm{~mm}$ ) and preserved systolic function, so he was initially medically managed. After 4 months of follow up, he had aortic valve replacement because of AR severity progression without 
Citation: Aureo Campos-Tello, Giancarlo A Valle, Félix A Revilla, et al. An infrequent association: Left ventricular non compaction with bicuspid aortic valve and coarctation of the aorta. Curr Trend Cardiol 2020;4(1):5-8.

variations in LV diameter or ejection fraction. The outcome was favorable [7].

An even more rare association is the one described for the first time by Myers et al:: a 15-year-old female with LVNC, BAV and coarctation of the aorta. She also had a subaortic VSD. Thus, the LV had severely enlargement and impairment of ejection fraction. This complex was called by the authors as Laubry-Pezzi syndrome [8].

Our patient had the association of LVNC, bicuspid aortic valve with severe regurgitation and coarctation of the aorta. None of the above cases mentioned before had a patient with these three features. Despite the severity of aortic regurgitation and mild LV enlargement (measured by TTE and CMR), the patient had a NYHA I functional class with preserved LVEF; thus, we decided medical therapy. This conservative approach was the same applied initially by Wrigley et al. [7].

Even in presence of bicuspid aortic valve and severe aortic regurgitation, we decided for a conservative strategy because, according to the 2017 European Society of Cardiology Guidelines for the management of valvular heart disease, a patient with severe AR should undergo surgery if symptomatic, LVEF $<50 \%$, LVEDD $>70 \mathrm{~mm}$ or LVESD $>50 \mathrm{~mm}$ ); our patient didn't met none of this criteria. It's also known that a patient with severe AR should undergo surgery if it's planned an intervention of ascending aorta, but our patient didn't have that indication either. This happens because in presence of bicuspid aortic valve, a patient should be scheduled for surgery if the maximal ascending aortic diameter $\geq 50 \mathrm{~mm}$ with risk factor or $\geq 55 \mathrm{~mm}$ without them; our patient didn't reach those cutoffs (he had $42 \mathrm{~mm}$ at the sinuses of Valsalva level) [9].

Finally, the coarctation of the aorta had class I indication for intervention due to $\mathrm{BP}>140 / 90 \mathrm{mmHg}(145 / 91 \mathrm{mmHg}), \mathrm{BP}$ difference $>20 \mathrm{mmHg}$ between upper and lower limbs (in our case was $45 \mathrm{mmHg}$ ) and significant LV hypertrophy detected by TTE (IVSd: $13.5 \mathrm{~mm}$ ) [10]. Therefore, we decided for percutaneous intervention of the coarctation, medical therapy and clinical-imaging follow up. The patient received Enalapril $5 \mathrm{mg}$ PO BID and Furosemide $40 \mathrm{mg}$ PO QD. By now, he continues with that treatment, remains intervention.

\section{Conclusion}

The patient has certain features not reported before in current literature. That's why we decided to publish this case. We're completely sure that if this patient only had LVNC, he'll never had his disease detected because most of LVNC are asymptomatic. The associated abnormalities (BAV with AR and coarctation of the aorta) made possible to identify and diagnose this patient, since it all started with just a murmur. We should emphasize the importance of an accurate physical examination and non-invasive images that that played a crucial role in this case.

\section{References}

1. Feltes-Guzmán G, Nunez-Gil I. Left ventricular non compaction. An article from the e-journal of the ESC Council for Cardiology Practice. European Society of Cardiology. 2012; 10(31):26.

2. Dong X, Fan P, Tian T, et al. Recent advancements in the molecular genetics of left ventricular noncompaction cardiomyopathy. Clin Chim Acta. 2017; 465:40-44.

3. Finsterer J, Stöllberger C, Towbin J. Left ventricular non compaction cardiomyopathy: Cardiac neuromuscular, and genetic factors. Nature Reviews Cardiology.2017; 14(4): 224-37.

4. Jacquier T, Thuny F, Jop B, et al. Measurement of trabeculated left ventricular mass using cardiac magnetic resonance imaging in the diagnosis of left ventricular noncompaction. European Heart Journal.2010; 31(9): 1098-1104.

5. Petersen SE, Selvanayagam JB, Wiesmann F, et al. Left ventricular non-compaction: Insights from cardiovascular magnetic resonance imaging. J Am Coll Cardiol. 2005; 46 (1):101-05.

6. Cavusoglu Y, Ata N, Timuralp B, et al. Noncompaction of the ventricular myocardium: Report of two cases with bicuspid aortic valve demonstrating poor prognosis and with prominent right ventricular involvement. Echocardiography. 2003; 20(4):379-383.

7. Wrigley Benjamin J, Rosin M, Banerjee P. Replacement of a congenital bicuspid aortic valve in a patient with left ventricular noncompaction. Tex Heart Inst J. 2009; 36(3): 241-43.

8. Myers PO, Tissot C, Cikirikcioglu M, et al. Complex aortic coarctation, regurgitant bicuspid aortic valve with VSD and ventricular non-compaction: A challenging combination. Thorac Cardiov Surg. 2011; 59(5):313-16.

9. Baumgartner H, Falk V, Bax JJ, et al. 2017 ESC/EACTS Guidelines for the management of valvular heart disease. Eur Heart J. 2017; 38(36):2739-91.

10. Baumgartner H, Bonhoeffer P, De Groot NM, et al. ESC Guidelines for the management of grown-up congenital heart disease. Eur Heart J. 2010; 31(23):2915-57.

\section{Correspondence to:}

Aureo Campos-Tello

Department of Cardiology

Hospital Nacional Dos de Mayo

Lima

Peru

E-mail: afernandocampos@gmail.com 